\title{
Ciclos de vida de insectos dulceacuícolas y cambio climático global en la ecorregión subantártica de Magallanes: investigaciones ecológicas a largo plazo en el Parque Etnobotánico Omora, Reserva de Biosfera Cabo de Hornos (55 $\mathrm{S}$ )
}

\author{
Life cycles of freshwater invertebrates and global climate change in the sub-Antarctic Magellanic \\ ecoregion: long-term ecological research at the Omora Ethonobotanical Park, Biosphere Reserve \\ Cape Horn $\left(55^{\circ} \mathrm{S}\right)$
}

\author{
Tamara Contador a,b,c*, James Kennedy ${ }^{\text {a,c,d,f, Jaime Ojeda }}{ }^{\text {a,b,c }}$, Peter Feinsinger ${ }^{\text {f,g, }}$, Ricardo Rozzi ${ }^{\text {a,b,c,e }}$ \\ * Autor de correspondencia: a Universidad de Magallanes, Avenida Bulnes 01855, Punta Arenas, Chile, tamara.contador@umag.cl \\ ${ }^{\mathrm{b}}$ Instituto de Ecología y Biodiversidad, Las Palmera 3425, Ñuñoa, Santiago. \\ c Programa de Conservación Biocultural Subantártica, Parque Etnobotánico Omora LTSER. \\ ${ }^{\mathrm{d}}$ University of North Texas, Department of Biology Science, Denton, TX, USA.

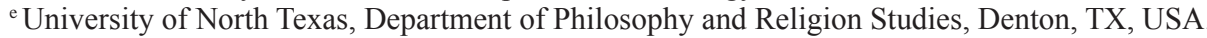 \\ ${ }^{\mathrm{f}}$ Northern Arizona University, Department of Biological Sciences, Flagstaff, Arizona, USA. \\ ${ }^{g}$ Latin American and Caribbean Program, Wildlife Conservation Society, Bronx, New York, USA.
}

\begin{abstract}
SUMMARY
The Omora Ehtonobotanical Park $\left(55^{\circ} \mathrm{S}\right)$ is the southernmost site of the LTSER network-Chile and the interdisciplinary research center of the Cape Horn Biosphere Reserve in the sub-Antarctic Magellanic ecoregion. The park protects the Róbalo River watershed that provides water to Puerto Williams, the capital of the Chilean Antarctic Province. In 2008, we initiated long-term studies on the diversity, distribution and life histories of aquatic insects associated with the Róbalo River and other streams on Navarino Island. These studies are of major interest to the LTSER-Chile network and to world science for three reasons: 1) the unique climatic characteristics of the sub-Antarctic Magellanic ecoregion, which contrast with those of the Northern Hemisphere; 2) responses of freshwater insects and their life cycles are very sensitive to temperature, and along the thermic variations associated with the altitudinal gradient of the Róbalo River we can make predictions for various scenarios of Global Climate Change; 3) the life cycles of freshwater insects have been understudied in southwestern South America, and by incorporating similar studies of phenological responses of freshwater insects at other sites LTSER-Chile sites along a latitudinal gradient will allow us to assess early signals of this biota to global climate change.
\end{abstract}

Key words: benthic macroinvertebrates, phenology, stream ecology, conservation.

\section{RESUMEN}

El Parque Etnobotánico Omora, ubicado en la ecorregión subantártica y en la Reserva de Biosfera Cabo de Hornos $\left(55^{\circ} \mathrm{S}\right)$ es el sitio más austral de la red LTSER-Chile. El Parque protege la cuenca hidrográfica del río Róbalo, que provee de agua a Puerto Williams, la capital de la Provincia Antártica Chilena. El año 2008, se inició un estudio a largo plazo sobre la diversidad, historias de vida y distribución de insectos acuáticos asociados al río Róbalo, y otros cursos de agua en la isla Navarino. Estos estudios son de gran interés para la red LTSER-Chile y la ciencia mundial por tres razones: 1) las características climáticas únicas de los ecosistemas dulceacuícolas de la ecorregión subantártica de Magallanes, que contrastan con aquellas del Hemisferio Norte; 2) las respuestas de los insectos dulceacuícolas y sus ciclos de vida son muy sensibles a la temperatura, y bajo condiciones de gradientes térmicos del gradiente altitudinal del río Róbalo se pueden hacer predicciones bajo distintos escenarios de Cambio Climático Global; y 3 ) los ciclos de vida de insectos dulceacuícolas han sido muy poco estudiados en el suroeste de Sudamérica, e incorporando el gradiente latitudinal con estudios similares de respuestas fenológicas de los insectos dulceacuícolas incorporando otros sitios de la Red LTSER-Chile permitiría evaluar señales tempranas de esta biota al cambio climático global.

Palabras clave: macroinvertebrados bentónicos, fenología, ecología de ríos, conservación.

\section{INTRODUCCIÓN}

La ecorregión subantártica de Magallanes ha sido identificada como una de las 24 zonas prístinas del planeta (Mittermeier et al. 2003) ya que: (i) conserva más del 70 \% de la vegetación original, (ii) abarca un área exten- sa mayor a $10.000 \mathrm{~km}^{2}$, (iii) posee una de las densidades poblacionales humanas más bajas dentro de las latitudes templadas y carece de desarrollo industrial significativo. La ecorregión se extiende desde el Cabo de Hornos $\left(56^{\circ} \mathrm{S}\right)$ hasta el Golfo de Penas ( $47^{\circ} \mathrm{S}$ ) (figura 1). Esta zona de archipiélagos contiene los bosques más australes del pla- 


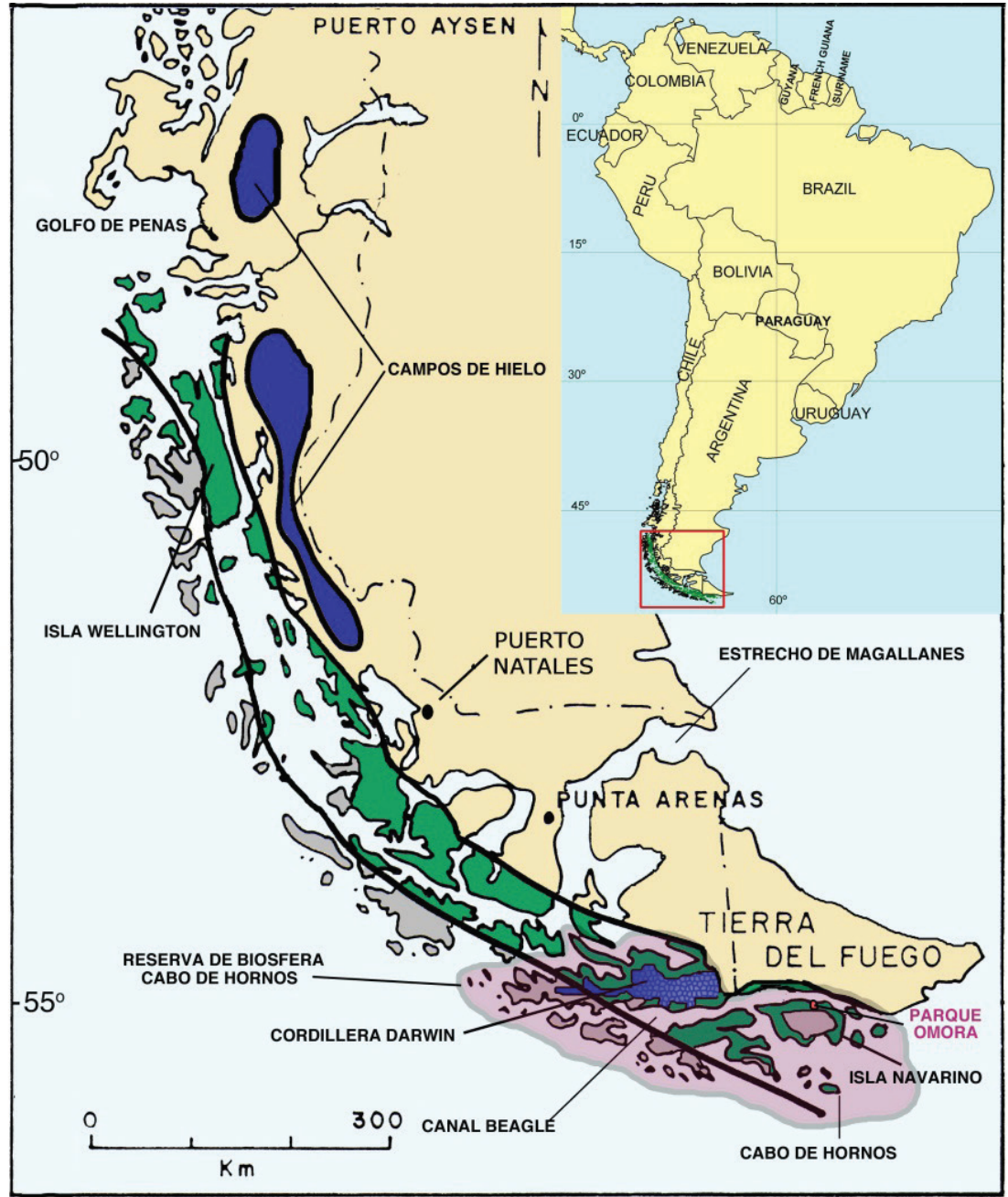

Figura 1. Ecorregión subantártica (o subpolar) de Magallanes, entre el Cabo de Hornos ( $56^{\circ} \mathrm{S}$ ) y el Golfo de Penas (47 $\left.\mathrm{S}\right)$. En color verde se indica la zona dominada por bosques subantárticos siempreverdes dominados por Nothofagus betuloides. En color gris se indica la zona dominada por el complejo de tundra de Magallanes. En color azul se indican las zonas dominadas por glaciares asociadas los campos de hielos. Se indica la ubicación del Parque Etnobotánico Omora en la isla de Navarino situada al sur de Tierra del Fuego, donde el 2008 se iniciaron los estudios a largo plazo sobre los ciclos de vida de los insectos dulceacuícolas. El Parque Omora constituye el sitio más austral de la red LTSER-Chile y el sitio de investigación, educación y conservación biocultural de la Reserva de Biosfera Cabo de Hornos, indicada por el color rosa pálido. Figura modificada de Rozzi et al. (2008).

The sub-Antarctic (or subpolar) Magellanic ecoregion, between Cape Horn ( $\left.56^{\circ} \mathrm{S}\right)$ and the Gulf of Penas ( $\left.47^{\circ} \mathrm{S}\right)$, showing the full extent of the evergreen forests dominated by Nothofagus betuloides (in green), the Magellanic moorland complex (in grey), and the ice fields (in blue). Located on Navarino Island south of Tierra del Fuego, is Omora Park, the southern most site of the LTSER-Chile network, and the biocultural research, education and conservation center of the Cape Horn Biosphere Reserve (indicated by light pink color). Figure modified Rozzi et al. (2008).

neta, en un ámbito latitudinal único en el mundo puesto que se extiende por diez grados de latitud más al sur que los ecosistemas boscosos más australes de Nueva Zelanda y Australia (Rozzi et al. 2012).

La ecorregión subantártica de Magallanes forma parte del bioma de los bosques templados de Sudamérica, extendiéndose por el sudoeste del continente hasta los $35^{\circ} \mathrm{S}$ (Armesto et al. 1998). Por lo tanto, el ámbito latitudinal del bioma abarca más de $20^{\circ}$ de latitud, lo que constituye el ámbito latitudinal de bosques templados más extenso en el Hemisferio Sur. Asociado al gradiente latitudinal, se presenta un marcado gradiente térmico, que ofrece un laboratorio natural ideal para investigar respuestas de la biota terrestre y dulceacuícola al cambio climático global. Además, el suroeste de Sudamérica se ubica fuera de las corrientes de aire que contienen contaminantes industriales y sus lluvias se originan en el sur del océano Pacífico. Por lo tanto, los bosques australes y sus ecosistemas están libres de la contaminación atmosférica; de hecho, estudios de la química de las aguas de lluvia en el bioma de los bosques templados de Sudamérica han revelado una de las concentraciones más bajas de nitrato registradas a nivel planetario 
(Likens 1991, Weathers et al. 2000, Galloway et al. 2004). Este escenario proporciona una oportunidad para investigar los efectos del cambio climático sobre la biota bajo condiciones que han experimentado un impacto antropogénico mucho menor al registrado en ecosistemas forestales templados del Hemisferio Norte (Hedin et al. 1995), permitiendo comenzar estudios de línea base para monitorear la calidad del agua de estos ecosistemas en el largo plazo utilizando macroinvertebrados bentónicos como indicadores.

En este artículo se ofrece una concisa síntesis de los estudios sobre la fenología en insectos dulceacuícolas desarrollados en el sitio más austral de la Red Chilena de Estudios Socio-Ecológicos a Largo Plazo (LTSER-Chile): el Parque Etnobotánico Omora en la Reserva de Biosfera Cabo de Hornos. El objetivo es presentar estos estudios y discutir tres puntos de alto interés para la red LTSER-Chile y la ciencia mundial: 1) las características climáticas únicas de los ecosistemas dulcecuícolas de la ecorregión subantártica de Magallanes; 2) las respuestas de los insectos dulceacuícolas y sus ciclos de vida bajo condiciones de gradientes térmicos desde la desembocadura hasta el nacimiento de cursos de agua en esta ecorregión; 3) la relevancia de implementar estudios similares de respuestas fenológicas de los insectos dulceacuícolas a lo largo de un gradiente latitudinal, incorporando otros sitios de la Red LTSER-Chile. Estos estudios son críticos para entender adecuadamente las diferencias en las respuestas de la biota al cambio climático global en el Hemisferio Sur, respecto a zonas latitudinalmente equivalentes en el Hemisferio Norte.

Nuestra investigación a largo plazo en el Parque Etnobotánico Omora también es relevante respecto a respuestas sociales que se pueden sugerir para confrontar el cambio climático global. La investigación abarca no sólo las respuestas de la biota, sino también estudios y acciones de educación, ecoturismo y toma de decisiones ambientales para orientar buenas prácticas que contribuyan a la conservación de la biodiversidad y la sustentabilidad socio-ecológica. Por un lado, en el contexto del cambio climático global los insectos dulceacuícolas pueden constituir especies centinela, que nos pueden entregar alertas tempranas de los efectos del cambio climático. Por otro lado, las metodologías que integran los estudios ecológicos y la ética ambiental en los estudios, los ecosistemas dulceacuícolas pueden generar acciones centinelas, que permitan a la red LTSER-Chile sugerir respuestas sociales para la sustentabilidad de la región y del planeta.

\section{PARQUE ETNOBOTÁNICO OMORA Y ESTUDIOS DE FENOLOGÍA DE INSECTOS DULCEACUÍCOLAS}

El año 2008 se iniciaron estudios sobre la diversidad, historias de vida y distribución de insectos acuáticos en el Parque Etnobotánico Omora (545ㅗ ' S; 67³9' O) en la isla Navarino. Esta isla se encuentra al sur de Tierra del Fuego y al norte del archipiélago del Cabo de Hornos (figura 1). El parque protege la cuenca hidrográfica del río Róbalo, el cual tiene una extensión de aproximadamente $12 \mathrm{~km}$, baja a través de un marcado gradiente altitudinal desde el cordón montañoso Dientes de Navarino y desemboca en el Canal Beagle. El Parque Omora cumple tres funciones principales: a) protección de la cuenca hidrográfica del río Róbalo que provee de agua potable a la población de Puerto Williams, capital de la Provincia Antártica Chilena y la ciudad más austral del mundo, b) conservación de la biodiversidad subantártica de Magallanes en un Sitio Prioritario para la Conservación de la Biodiversidad identificado por CONAMA el año 2002, c) sitio más austral y cofundador de la red LTSER-Chile, que funciona también como Centro de Visitantes y Estudios de la Reserva de Biosfera Cabo de Hornos y del Programa del Conservación Biocultural Subantártica.

Los estudios sobre insectos dulceacuícolas en el Parque Omora abordan cinco objetivos específicos: i) identificar las especies de insectos dulceacuícola del río Róbalo y otros cursos de agua en la Reserva de Biosfera Cabo de Hornos (RBCH); ii) monitorear a largo plazo la fenología de insectos acuáticos asociados al gradiente altitudinal del río Róbalo; iii) identificar especies de insectos acuáticos que puedan ser utilizados como indicadores del cambio climático global en la ecorregión subantártica de Magallanes; iv) contribuir a la protección de la cuenca hidrográfica del río Róbalo y de la biodiversidad de insectos dulceacuícolas en la $\mathrm{RBCH}$; y v) contribuir al desarrollo de programas de educación y actividades de turismo sustentable a través de la aproximación metodológica de la Filosofía Ambiental de Campo que integra los estudios ecológicos de los insectos acuáticos con la ética ambiental (Rozzi et al. 2010, Contador et al. 2011).

En enero del año 2008, a lo largo del gradiente altitudinal del río Róbalo, se establecieron cinco estaciones permanentes de muestreo, ubicadas a 120, 240, 386, 486, y 586 m s.n.m. (figura 2). Cada estación es representativa de uno de los cinco tipos de comunidades vegetales más importantes en el perfil altitudinal (Contador 2011). Las tres primeras estaciones se ubican dentro de la zona de bosques subantárticos $(0-400 \mathrm{~m})$ : siempreverdes (Nothofagus betuloides (Mirb.) Oerst 1871) en la estación a $120 \mathrm{~m}$, bosques mixtos siempreverdes - deciduos (Nothofagus pumilio (Poepp et Endl.) Krasse) a $240 \mathrm{~m}$ y bosques deciduos (N. pumilio y N. antarctica (G. Forster) Oerst) a $380 \mathrm{~m}$. Las otras dos estaciones se ubican por sobre el límite arbóreo (400 - $450 \mathrm{~m}$ en la isla Navarino) en zonas dominadas por plantas en cojín a $486 \mathrm{~m}$ y por líquenes y briófitas a 586 m (Méndez et al. 2013).

Para estudiar la composición de especies de insectos dulceacuícolas, su distribución y fenología, durante cada verano austral se han tomado muestras de macroinvertebrados bentónicos en cada estación, se identifican las especies y registran sus densidades (organismos $\mathrm{m}^{-2}$ ). Como especie indicadora de cambio climático global se ha seleccionado a Gigantodax rufescens (Diptera: Simuliidae). Para su estudio fenológico detallado, se tomaron muestras de macroinvertebrados bentónicos cada dos semanas entre agosto 2009 y julio 2010 (Contador 2011). Cada 


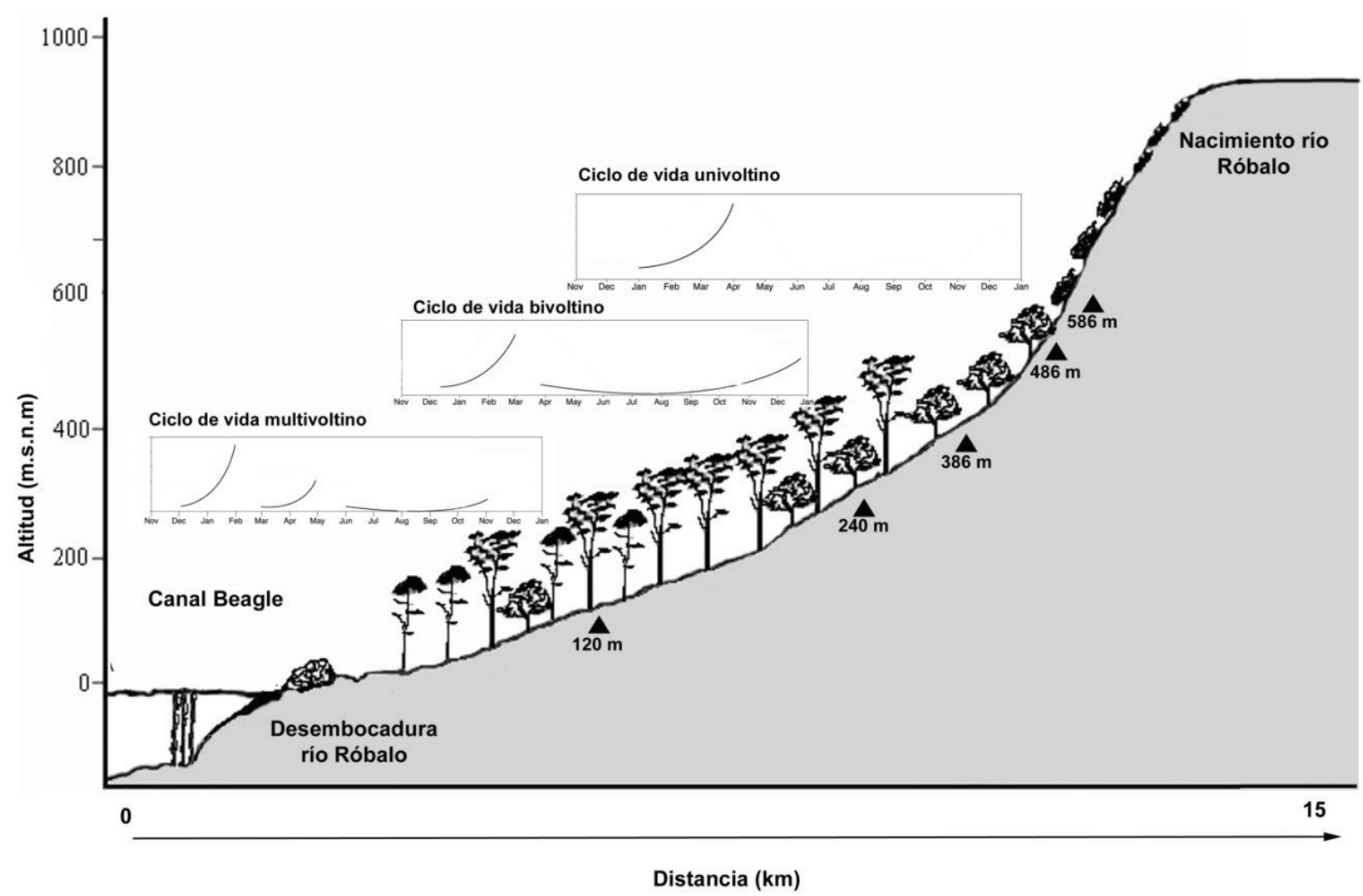

Figura 2. Gradiente altitudinal de la cuenca del río Róbalo en el Parque Omora, incluyendo los hábitats más representativos de la Reserva de Biosfera Cabo de Hornos. Desde la costa hacia las cumbres montañosas: bosques siempreverdes dominados por Nothofagus betuloides, bosques mixtos (siempreverdes y deciduos) dominados por $N$. betuloides y $N$. pumilio, bosques deciduos dominados por N. pumilio y N. antarctica, tundra altoandina dominada por plantas en cojín (Bolax gummifera) en las zonas bajas, y por musgos y líquenes (Neuropogon sp.) en las zonas más altas. Los triángulos indican la ubicación de cada una de las estaciones para estudios a largo plazo de insectos de dulceacuícolas. Los gráficos ilustran los ciclos de vida de Gigantodaxrufescens (Diptera: Simuliidae) en las estaciones de altitud inferior, media y superior (120, 386 y 586 m s.n.m.) que exhiben patrones multivoltinos, bivoltinos y univoltinos, respectivamente.

Altitudinal gradient of the Róbalo River watershed at Omora Park, including the most representative habitats of the Cape Horn Biosphere Reserve. From the coast to the mountain peaks: evergreen forests dominated by Nothofagus betuloides; mixed forests (evergreen and deciduous) dominated by $N$. betuloides and N. pumilio; deciduous forests dominated by N. pumilio y N. antarctica, high Andean tundra dominated by cushion plants (Bolax gummifera) in the lower areas, and by mosses and lichens (Neuropogon sp.) in the higher areas. The triangles indicate the location of each of the stations for long-term studies of freshwater insects. The graphs illustrate life cycles of Gigantodax rufescens (Diptera: Simuliidae) in the lower, middle, and higher altitude stations (120, 386 and $586 \mathrm{~m}$ a.s.1.) exhibiting multivoltine, bivoltine, and univoltine patterns, respectively.

estación está equipada con un monitor de temperatura (Data logger HOBO®, modelo U22 Water Temp ProV2) que registra la temperatura promedio, máxima y mínima diaria del agua desde el año 2008 hasta la fecha.

\section{CARACTERÍSTICAS CLIMÁTICAS DE LOS ECOSISTEMAS DULCEACUÍCOLAS SUBANTÁRTICOS DE MAGALLANES}

Los ecosistemas subantárticos del suroeste de Sudamérica contrastan marcadamente con sus pares latitudinales en el hemisferio Norte, principalmente por razones geográficas. En la banda latitudinal $40-60^{\circ} \mathrm{S}$ la proporción de superficie tierra: océano es $2 \%$ : $98 \%$; en cambio, en la franja latitudinal $40-60^{\circ} \mathrm{N}$ esta proporción es $2 \%$ : $98 \%$
(Rozzi et al. 2012). Esto genera un marcado contraste entre el macroclima de las zonas templadas y subpolares de ambos hemisferios. En el Hemisferio Sur, el clima en los ecosistemas templados y subpolares es modulado por la vasta extensión oceánica que determina un clima oceánico, caracterizado por una amplitud térmica anual baja con inviernos sin temperaturas frías extremas y veranos templados. En el Hemisferio Norte, el clima de los ecosistemas de latitudes altas es de tipo continental y se caracteriza por una amplitud térmica anual alta con inviernos muy fríos y veranos cálidos (Lawford et al. 1996). Por ejemplo, la amplitud térmica anual registrada por la estación microclimática de la Red LTSER-Chile Parque Etnobotánico Omora (10 m s.n.m.) es de $10,1^{\circ} \mathrm{C}$, obtenida como la diferencia entre la temperatura media del mes más cálido $\left(10,5^{\circ} \mathrm{C}\right.$, 
enero) y la temperatura media del mes más frío $\left(1,4^{\circ} \mathrm{C}\right.$, julio). En cambio, en el sitio LTER de Bonanza Creek en Alaska (6442'52” $\mathrm{N}$; $\left.148^{\circ} 08^{\prime} 43^{\prime \prime} \mathrm{O}\right)$, la amplitud térmica anual registrada es de $39,8^{\circ} \mathrm{C}$ (con temperaturas medias de $16,3{ }^{\circ} \mathrm{C} \mathrm{y}-23,5^{\circ} \mathrm{C}$ en los meses más cálidos (julio) y más fríos (julio), respectivamente.

El microclima a escala de cuencas y sus gradientes térmicos desde el nacimiento hasta la desembocadura de cursos de agua también presentaría diferencias inter-hemisféricas significativas. Las cuencas subantárticas de Magallanes se caracterizan por gradientes altitudinales con pendientes altas, un ámbito altitudinal bajo $(0-1.000 \mathrm{~m})$ y una topografía heterogénea donde se desarrolla un mosaico de turberas, bosques, matorrales y vegetación alto andina (Pisano 1977). Los datos de temperatura del agua registrados en el Parque Etnobotánico Omora sugieren que en la ecorregión subantártica de Magallanes, el efecto modulador de las temperaturas que ejerce el océano es fuerte cerca de las desembocaduras de los ríos, pero desparece drásticamente con la altitud, especialmente por sobre el umbral del límite arbóreo (Rozzi et al. 2010, Contador 2011) (figura 3). La temperatura promedio anual del agua del río Róbalo es $5,7^{\circ} \mathrm{C}$ a $120 \mathrm{~m}$ y de sólo $1,0^{\circ} \mathrm{C}$ a $586 \mathrm{~m}$. Es decir, la temperatura del agua es seis veces más fría en un corto gra- diente altitudinal $(<600 \mathrm{~m})$. Este resultado contrasta con ríos del hemisferio norte, donde un cambio térmico de esa magnitud se alcanza en intervalos altitudinales mucho mayores; por ejemplo, en aproximadamente a unos $1.500 \mathrm{~m}$ en las Montañas Rocallosas de Colorado en Norteamérica (Hauer et al. 1997).

El clima en el Parque Omora y la $\mathrm{RBCH}$ es afectado por los frentes polares del Pacífico del sur y por las trayectorias de las tormentas del oeste, acopladas por las precipitaciones inducidas por los vientos de la Cordillera de Los Andes y la Cordillera Darwin (McCulloch et al. 1997, Rozzi et al. 2006, Aravena y Luckman 2008). En base a los registros de la estación microclimática de la Red LTSER-Chile Parque Omora, instalada a 10 m s.n.m., la temperatura promedio anual del aire es de $5,7^{\circ} \mathrm{C}$, con una máxima promedio de $14,7{ }^{\circ} \mathrm{C}$ durante los meses cálidos (enero-marzo), y una mínima promedio de $-0,5^{\circ} \mathrm{C}$ durante los meses fríos (mayo-agosto). El Parque Omora mantiene otra estación meteorológica en la zona alto andina, ubicada en la ladera de exposición ecuatorial del cerro Bandera a 728 m s.n.m. (54 $\left.58^{\prime} 15,8^{\prime \prime} \mathrm{S} ; 67^{\circ} 38^{\prime} 01^{\prime \prime} \mathrm{O}\right)$, donde la temperatura media anual del aire es $0,02 \pm 4,41^{\circ} \mathrm{C}$, con una máxima promedio anual de $11,7 \pm 5,21{ }^{\circ} \mathrm{C}$ y una mínima promedio anual de $-6,18 \pm 2,86{ }^{\circ} \mathrm{C}$ (Méndez et al. 2013).

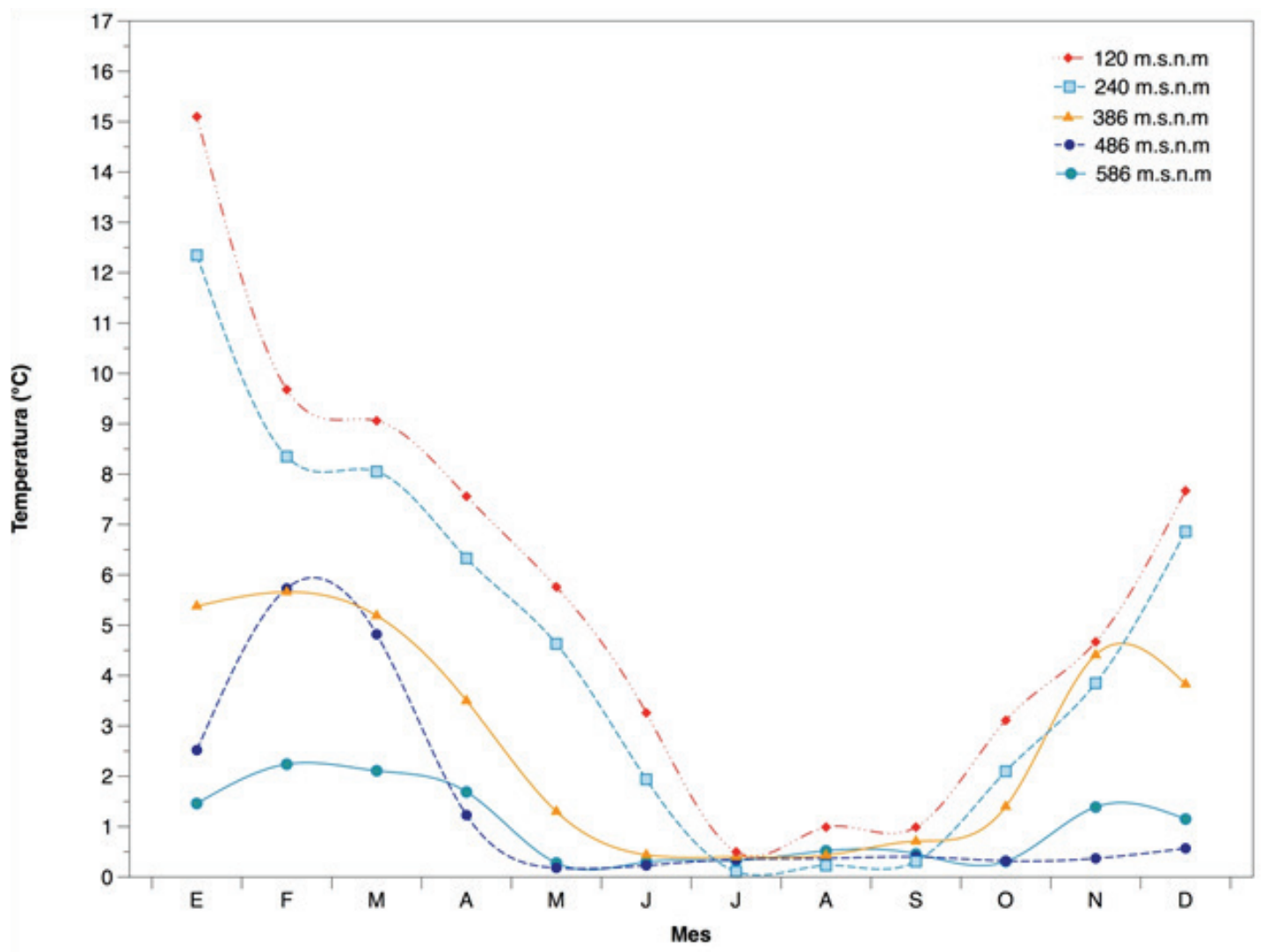

Figura 3. Temperatura promedio mensual en cada una de las estaciones permanentes para estudios a largo plazo de insectos dulceacuícolas, a lo largo del gradiente altitudinal del río Róbalo, Parque Omora $\left(55^{\circ} \mathrm{S}\right)$.

Average monthly temperature at each of the permanent stations for long-term studies of freshwater insects, along the altitudinal gradient of the Róbalo River, Omora Park (55 S). 


\section{RESPUESTAS DE LOS INSECTOS DULCEACUÍCOLAS A GRADIENTES TÉRMICOS ALTITUDINALES}

Uno de los factores más importantes en la distribución e historias de vida de insectos acuáticos a lo largo de gradientes de latitud y altitud es la temperatura del agua (Vannote et al. 1980). Para analizar la influencia de la temperatura sobre los ciclos de vida de los insectos dulceacuícolas, las medidas de temperatura se convierten a grados-días acumulados (GDA). La temperatura media anual del agua del río Róbalo genera $2051 \mathrm{GDA}\left(=5,7^{\circ} \mathrm{C}\right.$ x 365 días $)$ cerca de su desembocadura a $120 \mathrm{~m}$ y $365 \mathrm{DGA}\left(=1{ }^{\circ} \mathrm{C}\right.$ x 365 días) cerca de su nacimiento a $586 \mathrm{~m}$ (Contador 2011). Es decir, los grados-días acumulados (GDA) aumentan seis veces en un corto gradiente altitudinal $(<600 \mathrm{~m})$ (figura 3 ). Este marcado gradiente térmico genera variaciones en la fenología de los insectos acuáticos del río Róbalo. En la especie indicadora Gigantodax rufescens, se registró un ciclo de vida multivoltino con tres generaciones anuales (emergiendo en febrero, mayo y diciembre) en los pisos altitudinales bajos $(0-120 \mathrm{~m})$. A medida que aumenta la altitud, el número de generaciones anuales disminuye y en el nacimiento $(586 \mathrm{~m})$ este díptero presenta un ciclo de vida univoltino, con una sola generación de adultos emergiendo durante el mes de abril (figura 2). Los datos de temperatura sugieren que cada ciclo necesita 380 GDA para ser completado (Contador 2011).

Estos resultados obtenidos en el Parque Etnobotánico Omora contrastan con los obtenidos en latitudes similares del Hemisferio Norte, donde el desarrollo es lento y los casos de especies con más de una generación anual son excepcionales (Danks 2007). En latitudes menores $\left(34^{\circ} \mathrm{N}\right)$ las especies congenéricas presentan ciclos de vida frecuentemente multivoltinos. En el Hemisferio Norte, una gran cantidad de evidencia sugiere que los cambios en los ambientes térmicos a grandes escalas están afectando la fenología, desarrollo y actividad de insectos acuáticos en todos los niveles tróficos (Parmesan 2007, Richter et al. 2008). Además, los cambios vinculados a procesos metabólicos en la fenología de insectos acuáticos podrían afectar la sincronía de etapas clave en las historias de vida y la disponibilidad de recursos alimenticios o hábitats, provocando desacoples que podrían resultar en extinciones locales de poblaciones de insectos acuáticos (Menzel et al. 2006, Richter et al. 2008), generando cambios en la estructura de las comunidades acuáticas y en las distribuciones geográficas de ciertas especies (Windery Schindler 2004, Ryan y Ryan 2006, Woodward et al. 2010).

Los cambios climáticos ocasionan cambios múltiples en los ciclos de vida de los insectos acuáticos (Woodward et al. 2010), puesto que afectan no sólo su metabolismo, tasa crecimiento y desarrollo sino también su habilidad para sobrevivir en hábitats en donde las variaciones de temperatura exceden su rango de tolerancia térmica. Dado que la temperatura del agua varia espacialmente y temporalmente, la tolerancia térmica de los insectos acuáticos determina variaciones en su distribución (Ward y Stanford 1982, Woodward et al. 2010). Hasta la fecha en el río Róbalo se ha registrado un total de 42 taxones de macroinvertebrados bentónicos, que incluyen principalmente los órdenes de insectos Diptera, Plecoptera, Trichoptera y Ephemeroptera. Para la mayoría de las especies se han registrado patrones de distribución limitados en el gradiente altitudinal. Por ejemplo, el díptero Edwarsina sp. (Blephlariceridae) y el tricóptero Matigoptila brevicornuta Schmid. (Glossosomatidae) están restringidas a los pisos altitudinales bajos (0-200 m); en cambio, el plecóptero Udamocercia sp. (Notonemouridae) y el díptero Aphroteniella sp. (Chironomidae) se encuentran solamente en sitios cercanos al nacimiento del río (400-600 m).

\section{RELEVANCIA DE ESTUDIOS FENOLÓGICOS DE INSECTOS DULCEACUÍCOLAS EN LA RED LTSER-CHILE PARA CONFRONTAR EL CAMBIO CLIMÁTICO GLOBAL}

Las regiones polares subpolares, tanto subárticas como subantárticas, presentan evidencias particularmente fuertes de los efectos del cambio climático sobre comunidades de especies de agua dulce y sus ecosistemas (Sweeney et al. 1992, Spaulding et al. 2010). Varios modelos predicen un calentamiento dramático en ríos y arroyos, y en consecuencia la perdida de hábitats específicos para especies restringidas a bajas temperaturas (Winterbourn et al. 2008). Sin embargo, en el sur de Sudamérica los estudios sobre composición de especies fenología de insectos acuáticos son muy escasos (Contador et al. 2012). Uno de los pocos estudios ha sido realizado al este de la ecorregión subantártica de Magallanes, en la ecorregión de Patagonia por Beltran y Miserendino (2011), quienes registraron ciclos de vida semivoltinos y merovoltinos en los plecópteros Notoperla fasciata (Enderlein) y Notoperla magnaspina (Enderlein) (Gripopterygidae) en arroyos donde las temperaturas del agua variaban desde $1{ }^{\circ} \mathrm{C}$ hasta $12^{\circ} \mathrm{C}$. Estos registros contrastan con nuestros resultados, donde la presencia de ciclos de vida multivoltinos podría ser explicada por el clima oceánico en la isla Navarino (Danks 2007). Convey (1996) ha enfatizado que aunque en la ecorregión subantártica de Magallanes las temperaturas son relativamente bajas, no ocurren fríos extremos, lo que podría permitir un desarrollo continuo durante el año completo. Towns $(1980,1983)$ y Winterbourn (2008) han propuesto que las condiciones climáticas oceánicas del hemisferio sur podrían conducir a patrones de ciclos de vida en los insectos acuáticos diferentes a los documentados en el hemisferio norte. Los macroinvertebrados bentónicos que habitan en latitudes subpolares en el Hemisferio Norte $\left(>45^{\circ} \mathrm{N}\right)$ presentan ciclos de vida univoltinos, probablemente debido a las temperaturas extremas y la corta estación cálida que caracterizan el clima continental (Vannote y Sweeney 1980). En cambio el clima oceánico de las latitudes equivalentes del Hemisferio Sur, con temperaturas menos extremas y una estación cá- 
lida más extensa, podría explicar la presencia de ciclos de vida multivoltinos en altitudes bajas.

No sólo los ciclos de vida de los insectos acuáticos han sido poco estudiados en el suroeste de Sudamérica, sino también la diversidad de especies. De hecho, entre los continentes del mundo, Sudamérica presenta el porcentaje más bajo de publicaciones relacionadas al conocimiento y conservación de insectos acuáticos, y dentro de Sudamérica la ecorregión subantártica de Magallanes es la menos estudiada (Contador et al. 2012). Es en este contexto que habita una diversa fauna de invertebrados dulceacuícolas que sin duda incluye a números significativos de especies todavía desconocidas (Contador et al. 2012). En Chile, se conocen aproximadamente unas 1.000 especies de invertebrados dulceacuícolas, pero estudios sugieren que el número de especies todavía desconocidas en nuestro país podría incrementar significativamente la cifra (Valdovinos 2006).

\section{PASOS FUTUROS}

Un primer paso, en base a los antecedentes presentados, es consolidar los estudios de insectos dulceacuícolas en la cuenca hidrográfica del río Róbalo en el Parque Etnobotánico Omora. Este parque constituye un sitio ideal en la red LTSER-Chile para monitorear y eventualmente alertar tempranamente sobre efectos del cambio climático global sobre la fauna dulceacuícola. Los ecosistemas dulceacuícolas pueden ser considerados como ecosistemas centinela puesto que nos pueden entregar alertas tempranas y efectivas sobre los efectos del cambio climático a grandes escalas, y para esta función el Parque Omora representa un sitio crítico, ubicado en el extremo austral del continente americano.

En segundo lugar, se propone iniciar comparaciones latitudinales con respecto a la fenología y composición de insectos acuáticos a lo largo de un gradiente latitudinal dentro de la red LTSER-Chile, puesto que los ecosistemas de agua dulce están experimentando algunas de las tasas de calentamiento más rápidas del planeta (Hassan et al. 2005). Respecto a la composición y distribución de especies nuestras preguntas futuras incluyen: (1) ¿Qué tan cerca se encuentran ciertas especies de sus límites de tolerancia térmica? (2) ¿Cuál es su capacidad de adaptación o de aclimatación a los cambios? (3) ¿Qué cambios se observarán en las historias de vida, patrones de crecimiento y sobrevivencia dentro de una población que experimenta cambios en las temperaturas? y (4) ¿Cómo cambiarán las distribuciones de ciertas poblaciones a lo largo del espacio, especialmente, en gradientes altitudinales y latitudinales? (Stillman 2003). El estudio de especies con distribuciones restringidas y/o límites térmicos sensibles nos puede entregar información sobre los efectos del cambio climático no sólo en estos sitios, sino que también nos permitiría extrapolar sus efectos a regiones más cálidas del planeta (Woodward et al. 2010). Respecto al monitoreo del cambio climático en cursos de agua en otros sitios de la red LTSER-Chile, se considera que Gigantodax rufescens es una especie indicadora adecuada puesto que: i) tiene un ámbito de distribución amplio que se extiende desde la provincia del Aconcagua hasta el Cabo de Hornos (Wycodzinsky y Coscorón 1989); ii) es abundante en ríos y arroyos; iii) se encuentra en altitudes que varían desde el nivel del mar hasta el altoandino; iv) su ciclo de vida es sensible a cambios térmicos, variando de univoltino a multivoltino a través del gradiente altitudinal del río Róbalo. Debido a su distribución, se podría evaluar la fenología de esta especie en Senda Darwin LTSER $\left(42^{\circ} \mathrm{S}\right)$ y evaluar cambios que podrían generarse por al calentamiento global en el corto y largo plazo.

En tercer lugar, la investigación a largo plazo en el Parque Omora abarca estudios y acciones de educación formal y no-formal que han adaptado la metodología de la filosofía ambiental de campo para incluir el estudio y valoración a los invertebrados de agua dulce. Como resultado se ha creado una actividad innovadora en ecoturismo, Sumergidos con Lupa (Contador 2011). Esta actividad se podría implementar en otros sitios de la Red LTSER-Chile con el fin de fomentar el conocimiento y la valoración ecológica, económica, estética y ética de la fauna de invertebrados y ecosistemas dulceacuícolas por parte de sociedad.

En síntesis, bajo la aproximación de estudios a largo plazo del Parque Etnobotánico Omora, y en el contexto del cambio climático global, los insectos dulceacuícolas pueden constituir especies centinela que nos pueden entregar alertas tempranas de los efectos del cambio climático. Por otro lado, "sumergidos con lupa" y otras metodologías que integren la ecología y la ética ambiental en los estudios de los ecosistemas dulceacuícolas pueden generar acciones centinelas que permitan a la red LTSER-Chile sugerir respuestas sociales para la sustentabilidad de la región y del planeta.

\section{AGRADECIMIENTOS}

Agradecemos especialmente el apoyo de Francisco Olivares, Fernando Saldivia, Felipe Leyton y Carlos Saavedra, estudiantes del Liceo Donald McIntyre Griffits de Puerto Williams, quienes han colaborado con las investigaciones llevadas a cabo desde el año 2010. Agradecemos el apoyo de los estudiantes que han participado en el curso Tracing Darwin's Path, dictado por la University of North Texas y que se lleva a cabo en el Parque Etnobotánico Omora desde el año 2007, especialmente a Sebastian Rosenfeld, Heather Perry, Jeffrey Mabe, Ernesto Davis, Cristobal Pizarro, Rodrigo Molina, Kelli Moses, Alexandria Poole, Charles Braman y Michael Simononok, además del apoyo de Christopher Anderson durante el diseño de parte de los estudios. Agradecemos a Leopoldo Sancho, José Manuel Blanquer y Andrés Stambuk por colaborar en la toma de datos meteorológicos en las estaciones climáticas asociadas al Parque Omora. Tamara Contador es apoyada por el proyecto FONDECYT 11130451 y por el Proyecto FIC-R-201230127802 
y Peter Feinsinger por el proyecto CONICYT MEC80122013. El programa de investigación de insectos dulceacuícolas del Parque Etnobotánico Omora, es apoyado por la Universidad de Magallanes, el Instituto de Ecología y Biodiversidad (ICM, P05-002 y CONICYT PFB-23) y el Programa de Conservación Biocultural Subantártica, desarrollado en colaboración con University of North Texas.

\section{REFERENCIAS}

Aravena JC, Luckman BH. 2008. Spatio temporal rainfall patterns in southern South America. International Journal of Climatology. 29: 2106-2120. Disponible en http://www. interscience.wiley.com, DOI: 10.1002/joc.1761.

Beltran L, Miserendino, ML. 2011. Life cycle, production and habitat selection of Notoperla fasciata and N. magnaspina (Plecoptera: Gripopterygidae) in a headwater Patagonian Stream. Fundamental and Applied Limnology 178: 219-229.

Contador TA, Kennedy JH, Rozzi R. 2012. The conservation status of southern South American aquatic insects in the literature. Biodiversity and Conservation 21(8): 2095-2107.

Contador TA. 2011. Benthic macroinvertebrates of temperate, sub-Antarctic streams: the effects of altitudinal zoning and temperature on the phenology of aquatic insects associated to the Róbalo river, Navarino Island ( $\left.55^{\circ} \mathrm{S}\right)$, Chile. University of North Texas. Denton, Texas, USA. Tesis Doctoral. Department of Biological Sciences, University of North Texas. 140 p.

Convey P. 1996. Overwintering strategies of terrestrial invertebrates in Antarctica - the significance of flexibility in extremely seasonal environments. European Journal of Entomology 93: 489-505.

Danks HV. 2007. How aquatic insects live in cold climates. The Canadian Entomologist 139: 443-471.

Hauer RF, JS Baron, DH Campbell, KD Fausch, SW Hostetler, GH Leavesley, PR Leavitt, DM McKnight, JA Stanford. 1997. Assessment of climate change and freshwater ecosystems of the Rocky Mountains, USA and Canada. Hydrological Processes 11: 903-924.

Hedin LO, JJ Armesto, AH Johnson. 1995. Patterns of nutrient from unpolluted, old-growth temperate forest: evaluation of biogeochemical theory. Ecology 76: 493-509.

Lawford RG, PB Alaback, E Fuentes eds. 1996. High-Latitude Rainforests and Associated Ecosystems of the West Coast of the Americas: Climate, Hydrology, Ecology, and Conservation. New York, USA. Springer. 413 p.

Likens GE. 1991. Some consequences of long-term human impacts on ecosystems. Revista Chilena de Historia Natural 64: 597-614.

McCulloch RD, CM Clapperton, J Rabassa, AP Currant. 1997. The glacial and post-glacial environmental history of Fuego-Patagonia. In McEwan C, LA Borrero, A Prieto eds. Patagonia: Natural History, Prehistory and Ethnography. New Jersey, USA. Princeton. p. 12-31.

Méndez M, R Rozzi, L Cavieres. 2013. Flora vascular y no-vascular en la zona altoandina de la isla Navarino $\left(55^{\circ} \mathrm{S}\right)$, Reserva de biosfera Cabo de Hornos, Chile. Gayana Botanica 70(2): 337-343.

Menzel A, TH Sparks, N Estrella, E Koch, A Aasa, R Ahas, K Alm-Kübler. 2006. European phenological response to cli- mate change matches the warming pattern. Global Change Biology 12(10): 1969-1976.

Mittermeier RA, CG Mittermeier, TM Brooks, JD Pilgrim, WR Konstant, GAB da Fonseca, C Kormos. 2003. Wilderness and Biodiversity Conservation. Proceedings of the National Academy of Sciences 100: 10309-10313.

Parmesan C. 2007. Influences of species, latitudes and methodologies on estimates of phenological response to global warming. Global Change Biology 13(9): 1860-1872.

Pisano E. 1977. Fitogeografía de Fuego-Patagonia Chilena I. Comunidades vegetales entre las latitudes 52 y $56 \mathrm{~S}$. Anales del Instituto de la Patagonia 8: 121-250.

Richter O, F Suhling, O Müller, D Kern. 2008. A model for predicting the emergence of dragonflies in a changing climate. Freshwater Biology 53(9): 1868-1880.

Rozzi R, F Massardo, C Anderson, K Heidinger, J Silander. 2006. Ten Principles for Biocultural Conservation at the Southern Tip of the Americas: The Approach of the Omora Ethnobotanical Park. Ecology \& Society 11(1): 43.

Rozzi R, CB Anderson, JC Pizarro, F Massardo, Y Medina, A Mansilla, JH Kennedy, J Ojeda, TA Contador, V Morales, K Moses, A Poole, JJ Armesto, MT Kalin. 2010. Field environmental philosophy and biocultural conservation at the Omora Ethnobotanical Park: Methodological approaches to broaden the ways of integrating the social component (S) in Long-Term Socio-Ecological Research (LTSER) Sites. Revista Chilena de Historia Natural 83: 27-68.

Rozzi R, JJ Armesto, JR Gutiérrez, F Massardo, GE Likens, CB Anderson, A Poole, K Moses, G Hargrove, A Mansilla, JH Kennedy, M Wilson, K Jax, C Jones, BJ Callicott, M Arroyo. 2012. Integrating Ecology and Environmental Ethics: Earth Stewardship in the Southern End of the Americas. BioScience 62(3): 226-236.

Ryan PA, AP Ryan. 2006. Impacts of global warming on New Zealand freshwater organisms: a preview and review. New Zealand Natural Sciences 31: 43-57.

Spaulding SA, B Van, DE Vuver, DA Hodgson, DM Mcknight, E Verleyen. 2010. Diatoms as indicators of environmental change in Antarctic and subantarctic freshwaters. The Diatoms: Applications for the environmental and Earth sciences. New York, USA. Cambridge University Press. p. 267-283.

Stillman JH. 2003. Acclimation capacity underlies susceptibility to climate change. Science 301: 65.

Sweeney BW, JK Jackson, DJ Newbold, DH Funk. 1992. Climate Change and the Life Histories and Biogeography of Aquatic Insects in Eastern North America. In Firth P, SG Fisher eds. Global Climate Change and Freshwater Ecosystems. New York, USA. Springer. p. 144-176.

Towns DR. 1980. Life histories of benthic invertebrates in a Kauri Forest stream in Northern New Zealand. Australian Journal of Marine and Freshwater Resources 32: 191: 211.

Towns DR. 1983. Life history patterns of six sympatric species of Leptophlebiidae (Ephemeroptear) in a New Zealand stream and the role of interspecific competition in their evolution. Hydrobiologia 99: 37-50.

Vannote R, B Sweeney. 1980. Geographic Analysis of Thermal Equilibria : A Conceptual Model for Evaluating the Effect of Natural and Modified Thermal Regimes on Aquatic Insect Communities. The American Naturalist 115(5): 667-695.

Ward JV, J Stanford. 1982. The thermal responses in the evolutionary ecology of aquatic insects. Annual Review of Ento- 
mology 27: 97-117.

Winder M, D Schindler. 2004. Climate change uncouples trophic interactions in an aquatic ecosystem. Ecology 85(8): 21002106.

Winterbourn MJ, S Cadbury, C Ilg, AM Milner. 2008. Mayfly production in a New Zealand glacial stream and the potential effect of climate change. Hydrobiologia 603: 211-219.

Weathers KC, GM Lovett, GE Likens, NFM Caraco. 2000.
Cloudwater inputs of nitrogen to forest ecosystems in southern Chile: Forms, fluxes, and sources. Ecosystems 3: 590-595.

Woodward G, DM Perkins, LE Brown. 2010. Climate change and freshwater ecosystems: impacts across multiple levels of organization. Philosophical Transactions of the Royal Society of London. Series B, Biological Sciences 365(1549): 2093-106.

Recibido: 10.07.14

Aceptado: 14.10 .14 
